\title{
Time Series Predictive Analysis based on Hybridization of Meta-heuristic Algorithms
}

\author{
Zuriani Mustaffa ${ }^{\# 1}$, Mohd Herwan Sulaiman ${ }^{*}$, Dede Rohidin ${ }^{1}$, Ferda Ernawan ${ }^{\#}$, Shahreen Kasim ${ }^{2}$ \\ ${ }^{\#}$ Faculty of Computer Systems \& Software Engineering, Universiti Malaysia Pahang, 26300 Gambang, Pahang, Malaysia \\ e-mail: zuriani@ump.edu.my; ferda@ump.edu.my
}

*Faculty of Electrical \& Electronics Engineering, Universiti Malaysia Pahang, 26600 Pekan, Pahang, Malaysia. e-mail: mherwan@ieee.org

${ }^{1}$ School of Computing, Telkom University, 40257 Bandung, West Java, Indonesia

${ }^{2}$ Faculty of Computer Science and Information Technology, Universiti Tun Hussein Onn Malaysia, Batu Pahat, 86400, Malaysia

\begin{abstract}
This paper presents a comparative study which involved five hybrid meta-heuristic methods to predict the weather five days in advance. The identified meta-heuristic methods namely Moth-flame Optimization (MFO), Cuckoo Search algorithm (CSA), Artificial Bee Colony (ABC), Firefly Algorithm (FA) and Differential Evolution (DE) are individually hybridized with a well-known machine learning technique namely Least Squares Support Vector Machines (LS-SVM). For experimental purposes, a total of 6 independent inputs are considered which were collected based on daily weather data. The efficiency of the MFO-LSSVM, CSLSSVM, ABC-LSSVM, FA-LSSVM, and DE-LSSVM was quantitatively analyzed based on Theil's U and Root Mean Square Percentage Error. Overall, the experimental results demonstrate a good rival among the identified methods. However, the superiority goes to FA-LSSVM which was able to record lower error rates in prediction. The proposed prediction model could benefit many parties in continuity planning daily activities.
\end{abstract}

Keywords - computational intelligence; least squares support vector machines; machine learning; meta-heuristic; optimization; swarm intelligence; time series prediction

\section{INTRODUCTION}

Computational Intelligence (CI) algorithms have been extensively studied and used by researchers in addressing various real-life problems. It is becoming increasingly difficult to deny the efficiency of CI algorithms which includes machine learning and optimization techniques. These are proven by a good number of applications of these algorithms in the literature [1]-[3]. Since decades ago, the emerging of population-based optimization algorithms is undeniably encouraging. Among them are the well-known Genetic Algorithm (GA) [4] which its efficiency is undoubted.

Interestingly, since its invention, the GA is continuously studied and being a favorite in the academic community of interest. Years later, researchers started to pay attention to nature, where one after another nature-inspired optimization algorithms began to rise such as Particle Swarm Optimization (PSO) [5], Ant Coloy Optimization (ACO) ) [6]
Grey Wolf Optimizer (GWO) [7] and many others. These optimization algorithms which motivated based on natureinspired concept deal with choosing the best alternative related to the provided objective function [8]. As an optimizer, these algorithms can be seen not only used as a single algorithm but also hybrid with another algorithm, such as machine learning technique namely Support Vector Machines (SVM), Artificial Neural Network (ANN) to name a few. A considerable amount of CI-based approaches has been widely presented in order to address many complex real-world issues, including prediction, has been published in the literature. Prediction model based on CI was surveyed which focused on prostate cancer [9]. In the study, cancer data of different modalities are considered. Meanwhile, a hybrid algorithm which is based on Support Vector Regression (SVR) and PSO was presented in [10] for a month ahead of the residential sector's electricity demand. The simulations results showed that the presented method was superior compared to Artificial Neural Network (ANN), Auto-Regressive Integrated Moving Average (ARIMA), 
Multiple Linear Regressions and a few other techniques reported in the literature. Progressing further, a study in [11] proposed kernel extreme learning machine based on MFO which is applied in medical diagnoses namely Parkinson and breast cancer cases. The findings of the study demonstrate that the proposed method can serve as an effective decisionmaking method for the case under study. Meanwhile, in 2014, a hybrid Support Vector Machine (SVM) optimized by PSO has been presented by [12] for real estate price prediction. In the study, the PSO is utilized as an optimization tool to optimize the SVM parameters automatically.

In this paper, hybrid predictive modeling of LSSVM with five optimization algorithms namely Moth-flame Optimization (MFO), Cuckoo Search Algorithm (CSA), Artificial Bee Colony (ABC), Firefly Algorithm (FA) and Differential Evolution (DE) is presented which is realized in weather prediction. The MFO, CSA, ABC, FA, and DE are employed individually as an optimizer to LSSVM hyperparameters, which later are known as MFO-LSSVM, CSALSSVM, ABC-LSSVM, FA-LSSVM, and DE-LSSVM respectively. By employing LSSVM, one of the critical problems that need serious attention is that the generalization capability of LSSVM is highly reliant on the value sets to its hyper-parameters, namely regularization parameter and kernel parameter. Any inappropriate values set to these parameters would demote the prediction performance. These identified optimization algorithms are chosen due to several advantages which includes having a few tuning parameters [13] and its capability to address optimization problems. The identified hybrid algorithms are later realized on weather predictive analysis. It is well documented that weather prediction is vital as it would impact the lives of human being. Accurate weather prediction would help different types of businesses to plan for production, power consumption, and many other activities. Besides, it is also important for certain people who face health problems such as heat stress and asthma. By knowing the weather, proper plan and action can be taken to avoid unwanted situation to occur.

The remaining part of the paper structured as the followings: Section 2 describes on the fundamental theory of LSSVM, followed by Section 3 which presents the optimization of LSSVM based on the identified metaheuristic algorithms while the hybridization of LSSVM with those meta-heuristic algorithms are discussed in Section 4. Meanwhile, the implemented methodology is discussed in Section 5 while results are discussed and analyzed in Section 6 . Finally, Section 7 concludes the study.

\section{MATERIAL AND METHOD}

\section{A. Least Squares Support Vector Machines}

Introduced by Suykens and colleagues, LSSVM [14] is a new version of SVM which offers a better solution strategy with regards to solving the problem at hands. The improved version is proven to address the problem faced by SVM during training [15]. With the reformulation of LSSVM, it simplifies complex calculation which led to more comfortable and faster training task. With that, a simpler optimization problem can be obtained [16]. Also, LSSVM offers fewer control parameters, which are $\gamma$ and $\sigma^{2}$, compared to three control parameters required in SVM $(C$, $\sigma^{2,}$ and $\varepsilon$ ) [17]. In addition, regarding a prediction task, LSSVM is proven to be better than SVM [18].

Provided with a training set of $N$ points $\left\{x_{i}, y_{i}\right\}^{N}$ where $x_{i}$ and $y_{o u}$ both are the input and output values respectively, the goal is to estimate a model based on the given equation:

$$
y(x)=w^{T} \varphi\left(x_{i}\right)+b+e_{i}
$$

Where $w$ represents weight vector, $\varphi():. R^{n}$ is the non-linear function, $b$ indicates the bias and $e_{i}$ served as the error between the actual and predicted output at the $i$ th training data. $X_{i}$ and $y(x)$ act as input and output, respectively. The followings can obtain the coefficient vector $\mathrm{w}$ and bias term b:

$$
\min _{w, b, e} J(w, e)=\frac{1}{2} w^{T} w+\gamma \frac{1}{2} \sum_{i=1}^{N} e_{i}^{2}
$$

Subject to the equality constraints

$$
y_{i}=w^{T} \varphi\left(x_{i}\right)+b+e_{i}, i=1,2, \ldots, N
$$

By applying the Lagrangian multiplier to (2) produces:

$$
L(w, b, e, \alpha)=J(w, e)-\sum_{i=1}^{N} \alpha_{i}\left\{w^{T} \varphi\left(x_{i}\right)+b+e_{i}-y_{i}\right\}
$$

Where $\alpha \mathrm{i}$ are Lagrange multipliers and $\gamma$ is the regularization parameter. Differentiating (3) with w, b, ei and $\alpha \mathrm{i}$, the conditions for optimality is as express in the following:

$$
\begin{aligned}
& \frac{\partial L}{\partial w}=0 \rightarrow w=\sum_{i=1}^{N} \alpha_{i} \varphi\left(x_{i}\right) \\
& \frac{\partial L}{\partial b}=0 \rightarrow \sum_{i=1}^{N} \alpha_{i}=0 \quad i=1,2, \ldots, N \\
& \frac{\partial L}{\partial e_{i}}=0 \rightarrow \alpha_{i}=\gamma e_{i} \\
& \frac{\partial L}{\partial \alpha_{i}}=0 \rightarrow w^{T} \varphi\left(x_{i}\right)+b+e_{i}-y_{i}=0
\end{aligned}
$$

The following linear equations are obtained by eliminating the $\mathrm{w}$ and ei:

$$
\left[\begin{array}{cc}
0 & y^{\gamma} \\
y & \Omega+I / \gamma
\end{array}\right]\left[\begin{array}{l}
b \\
\alpha
\end{array}\right]=\left[\begin{array}{l}
0 \\
1_{v}
\end{array}\right]
$$

With $y=[y 1 ; \ldots ; y N], \alpha=[\alpha 1, \ldots, \alpha N], I$ is the identity matrix and $1 \mathrm{v}=[1 ; \ldots ; 1]$. The kernel trick is applied as follows:

$$
\begin{aligned}
& \Omega i l=\varphi\left(x_{i}\right)^{T} \varphi\left(x_{l}\right) \quad i, l=1, \ldots, N \\
& =K\left(x_{i}, x_{l}\right)
\end{aligned}
$$

The resulting of LSSVM model for regression in (1) becomes: 


$$
y(x)=\sum_{i=1}^{N} \alpha_{i} K\left(x, x_{i}\right)+b
$$

Where $\alpha$ and $b$ are the solutions of (5). Considering the non-linear features of the employed data set, in this study, Radial Basis Function (RBF) kernel is used as it is suitable to handle such data12 and able to give good performance in many prediction cases 13 . It is expressed as:

$$
y(x)=\sum_{i=1}^{N} \alpha_{i} K\left(x, x_{i}\right)+b
$$

Where $\sigma 2$ is one of the adjustable parameters which related to RBF kernel. The other adjustable parameter of $\mathrm{RBF}$ kernel is the regularization parameter, $\gamma$ which can be seen in (2).

In this study, the RBF kernel is employed considering its suitability in handling with nonlinear data [19]. Brief theory of LSSVM is presented here while a detailed description can be found in [14].

\section{B. Meta-Heuristic Algorithms}

This section is dedicated to present an optimization of LSSVM hyper-parameters based on identified meta-heuristic algorithms namely MFO, CSA, ABC, FA, and DE.

1) Moth-Flame Optimizer: Moth-flame optimization (MFO) [20] is a relatively recent nature-inspired optimization technique which is motivated by the uniqueness of moths' navigation method in nature. The navigation method is known as transverse orientation. They fly at night by maintaining a fixed angle concerning the moon where the moon is used to guide them in flying for long distances. Such guideline helps them in flying in a straight line. Nonetheless, moths are easily trapped in a deadly spiral path around artificial lights. The MFO algorithm was mathematically formulated based on this unique behavior for optimization purposes, in order to find the best possible solution(s) for a given problem. In this study, the capability of MFO is fully utilized as an optimizer to optimize the LSSVM hyper-parameters, which would increase the effectiveness of LSSVM in prediction. In the MFO algorithm, the successor solutions are represented by months while the position of the moths in space taking place the problem's variables. The moths can fly in various dimensions 1-D, 2D or even hyperdimensional space by changing their position vectors. The pseudo code of MFOLSSVM is shown in Fig. 1.

2) Cuckoo Search Algorithm: Cuckoo Search Algorithm (CSA) is one of the promising meta-heuristic algorithms which was invented by Xin-She Yang [21]. CSA is inspired by brood parasitism of cuckoo species, which is a main mechanism of cuckoos in nature. In CSA, the quality or fitness of a solution is modeled proportional to the objective function value. In CSA, the candidate solution indicates by each egg in the nest. Meanwhile, the new solution represents by the cuckoo's egg. The aim is to serve the new and potentially better solutions to replace a less good solution in the nests [8]. The pseudo code of CSA-LSSVM is shown in Fig. 2.

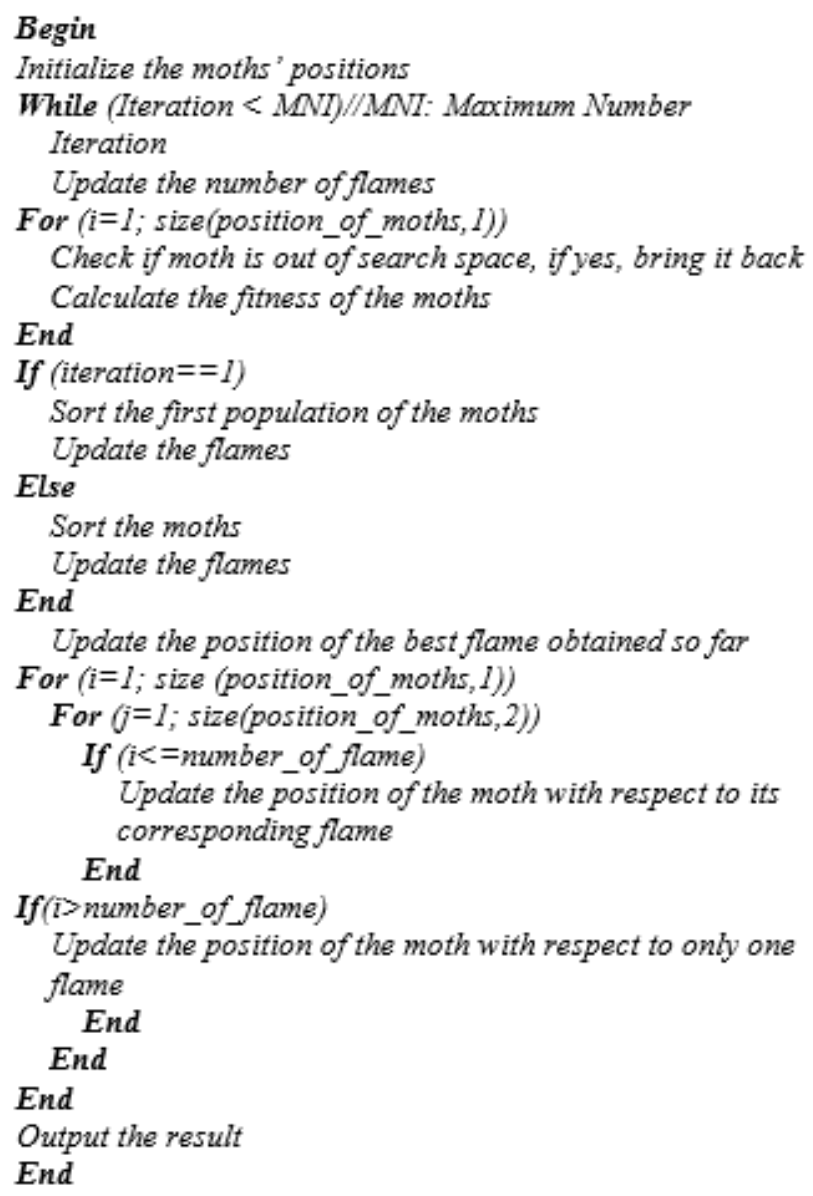

Fig. 1 Pseudo Code of MFO-LSSVM

3) Artificial Bee Colony Algorithm: The ABC algorithm [13] is developed from the observation of the brilliant foraging habits of honey bees swarm. In the algorithm, the honey bee swarm consists of three groups of bees. The first group is known as an employed bee, the second group is onlooker bees, and lastly, the third group is scout bees. In nature, all of the bees are working to maximize the amount of nectar. Fig. 3 shows the pseudo code of ABC-LSSVM.

4) Firefly Algorithm: social habits of fireflies inspire Firefly Algorithm (FA) [21]. In natural life of fireflies, the flashing features are employed to attract the mating partners. Meanwhile, the movement of fireflies is driven by the resulting attraction, and the attractiveness is related to the intensity of the emitted light.

The pseudo code of FA is shown in Fig. 4.

5) Differential Evolution: Unlike the other methods, DE is classified as an Evolutionary Algorithm, the same class as Genetic Algorithm. The natural selection mechanism motivates DE [22]. In DE, the candidate solutions possess an equal chance to be evaluated. Fig. 5 shows the pseudo code of DE-LSSVM. 


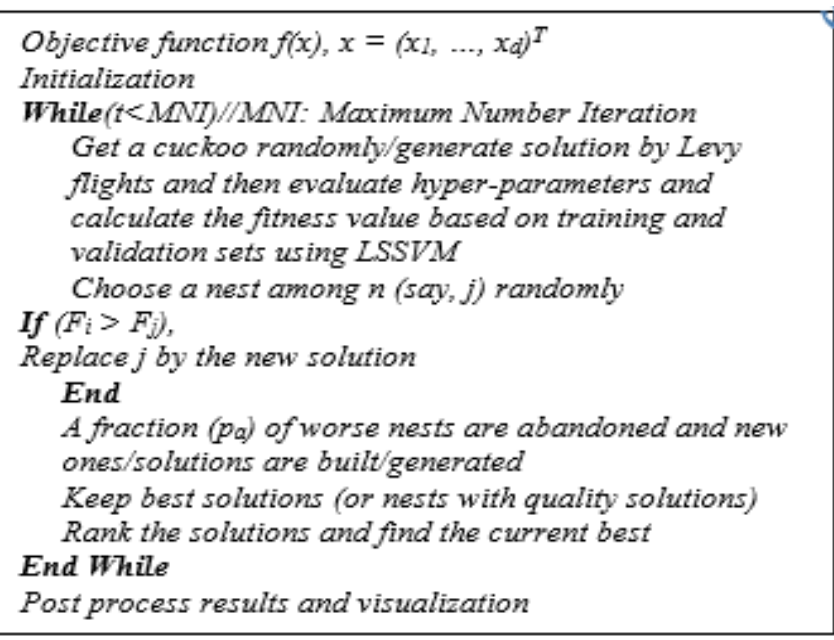

Fig. 2 Pseudo Code of CSA-LSSVM

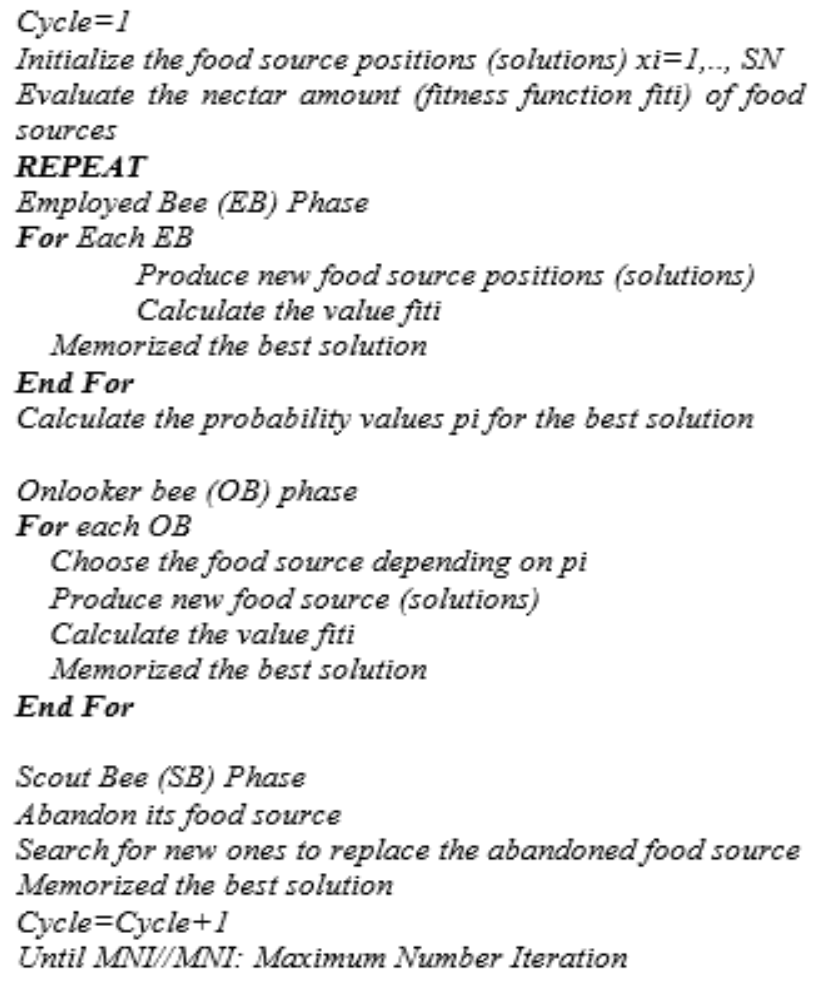

Fig. 3 Pseudo Code of ABC-LSSVM

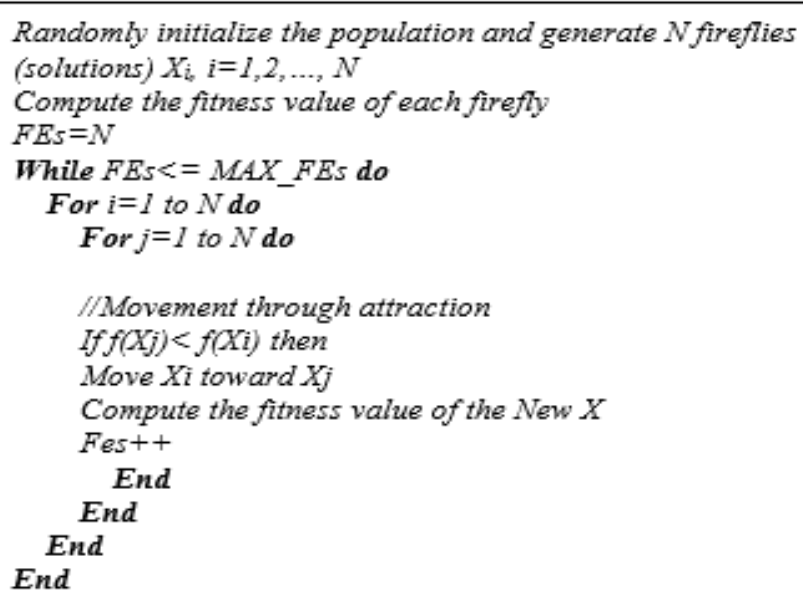

Fig. 4 Pseudo Code of FA-LSSVM

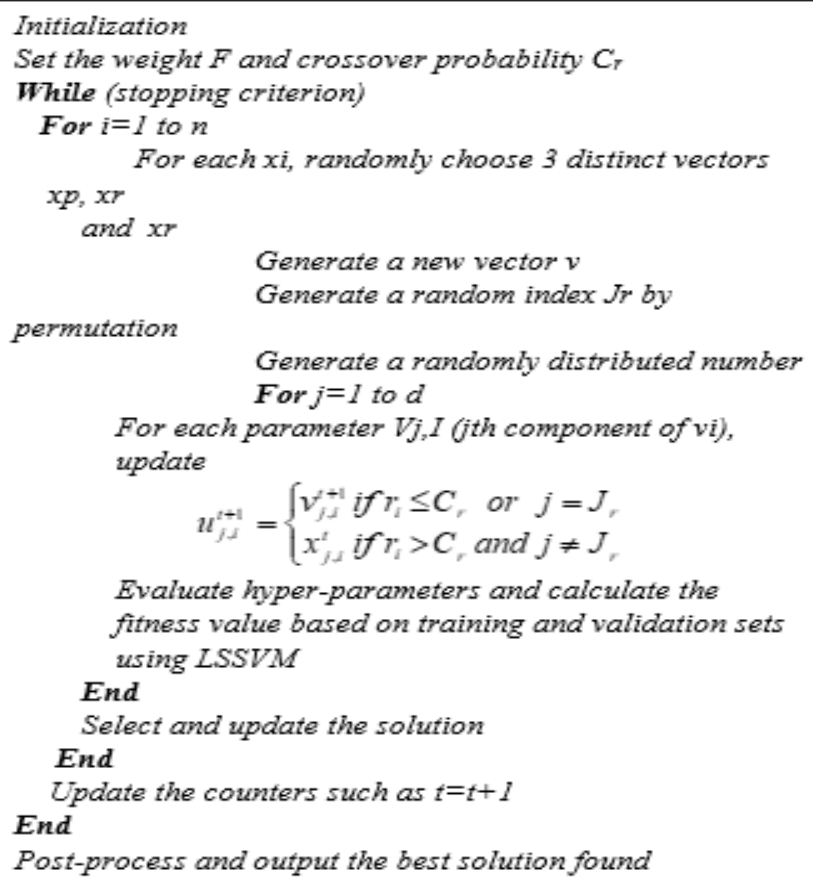

$$
u_{j-1}^{t+1}=\left\{\begin{array}{l}
v_{j-1}^{+1} \text { if } r_{i} \leq C_{r} \text { or } j=J_{r} \\
x_{j, j}^{t} \text { if } r_{i}>C_{r} \text { and } j \neq J_{r}
\end{array}\right.
$$

Evaluate hyper-parameters and calculate the fitness value based on training and validation sets

Fig. 5 Pseudo Code of DE-LSSVM

\section{Hybrid Meta-Heuristic with LSSVM Algorithm}

As briefly described in the previous section, five optimization algorithms are employed distinctively as an optimization tool to LSSVM to tune the hyper-parameters of LSSVM automatically; $\gamma$ and $\sigma^{2}$.

1) Initialization: Figure 6 shows the successor solution which are the two hyper-parameters of interest as illustrated in a matrix.

$$
X=\left[\begin{array}{cc}
\gamma 1 & \sigma^{2} 2 \\
\ldots & \ldots \\
\gamma 1 & \sigma^{2} 2
\end{array}\right] \quad \longleftarrow \quad \begin{aligned}
& \text { Candidate solution } 1 \\
& \text { Candidate solution } \ldots \\
& \text { Candidate solution } 20
\end{aligned}
$$

Fig. 6 Candidate Solution in $X$

In the proposed hybrid model, the LSSVM act in evaluating the fitness function and the optimal value of hyper-parameters of interest can be obtained after a termination criterion is hit. In this study, termination criterion s indicates by maximum number of iteration, which is set to 100 iteration. On the other hand, the objective function is guided by the selected statistical metric, Root Mean Square Error (RMSPE). The indicator of the RMSPE is, the smaller the value of the RMSPE, the better the result is.

2) Evaluation: In order to evaluate the objective function of the interested hyper-parameters, it is done based on training and validation set using LSSVM function, which is embedded in the five identified optimization algorithms. As describe previously, the objective function of the study is indicated by the selected statistical metric namely RMSPE.

The aim is to find an ideal combination of $\gamma$ and $\sigma^{2}$ which will result lowest RMSPE. 


\section{Experimental and performance criteria}

Discussion in this section focuses on the employed data, experimental setup and the utilized performance criteria.

1) Data Acquisition and Pre-Processing: For empirical Purposes, real-time series data that associated with temperature are employed. The data collected including high, average and low values of temperatures $\left({ }^{\circ} \mathrm{C}\right)$, dew points $\left({ }^{\circ} \mathrm{C}\right)$, humidity, sea level pressure $(\mathrm{hPa})$, visibility, winds $(\mathrm{km} / \mathrm{h})$ and precip $(\mathrm{mm})$. All data are obtained from www.wunderground.com. The data frequency is on daily basis which is recorded at Sultan Abdul Aziz Shah-Subang, Kuala Lumpur, Malaysia. This data set is ranging from April 2016 to May 2017. Samples of the data are tabulated in Table 1.

TABLE I

SAMPLES OF DATA

\begin{tabular}{|c|c|c|c|c|c|c|c|c|c|}
\hline $\begin{array}{c}\text { Date } \\
2016\end{array}$ & \multicolumn{2}{|c|}{ Temperature $\left({ }^{\circ} \mathrm{C}\right)$} & \multicolumn{3}{c|}{ Dew points $\left({ }^{\circ} \mathrm{C}\right)$} & \multicolumn{3}{c|}{ Humidity } \\
\cline { 2 - 10 } & High & Avg & Low & High & Avg & Low & High & Avg & Low \\
\hline $\begin{array}{c}\text { Apr } \\
2\end{array}$ & 35 & 30 & 26 & 25 & 24 & 23 & 89 & 72 & 49 \\
\hline $\begin{array}{c}\text { Apr } \\
3\end{array}$ & 36 & 31 & 25 & 27 & 24 & 22 & 89 & 71 & 44 \\
Apr & 36 & 30 & 25 & 24 & 23 & 20 & 94 & 69 & 41 \\
\hline $\begin{array}{c}4 \\
\text { Apr }\end{array}$ & 36 & 31 & 27 & 26 & 24 & 22 & 84 & 69 & 56 \\
\hline $\begin{array}{c}5 \\
\text { Apr }\end{array}$ & 34 & 29 & 25 & 25 & 24 & 23 & 94 & 78 & 59 \\
\hline
\end{tabular}

2) Data Normalization: For the sake of accuracy in prediction, the inputs and output will be normalized by using Zero Mean Normalization. The Zero Mean Normalization is defined as follow:

3) Input and Output Arrangement: Table 2 indicates the variables assigned to the features employed. For inputs, they are temperature, dew point, humidity, sea level pressure, visibility, wind speed, and precipitation. These inputs are fed to the prediction model to predict the temperature from day 5 ahead.

TABLE II

INPUT AND OUTPUT

\begin{tabular}{|c|c|c|c|}
\hline & Input & Variables & Output \\
\hline \multirow[t]{3}{*}{ Temperature $\left({ }^{\circ} \mathrm{C}\right)$} & Max & $\operatorname{maxT}$ & \multirow{19}{*}{$\begin{array}{c}\text { Temperature from } \\
\text { day } 5 \text { ahead } \\
\text { (temp5) }\end{array}$} \\
\hline & Mean & meant & \\
\hline & Min & $\min \mathrm{T}$ & \\
\hline \multirow[t]{3}{*}{ Dew Point $\left({ }^{\circ} \mathrm{C}\right)$} & $\operatorname{Max}$ & $\operatorname{maxDP}$ & \\
\hline & Mean & meanDP & \\
\hline & Min & $\operatorname{minDP}$ & \\
\hline \multirow[t]{3}{*}{ Humidity (\%) } & Max & $\operatorname{maxH}$ & \\
\hline & Mean & meanH & \\
\hline & Min & $\min \mathrm{H}$ & \\
\hline \multirow{3}{*}{$\begin{array}{c}\text { Sea level pressure } \\
(\mathrm{hPa})\end{array}$} & Max & $\operatorname{maxSLP}$ & \\
\hline & Mean & meanSLP & \\
\hline & Min & $\operatorname{minSLP}$ & \\
\hline \multirow[t]{3}{*}{ Visibility $(\mathrm{km})$} & Max & $\operatorname{maxV}$ & \\
\hline & Mean & meanV & \\
\hline & Min & $\min V$ & \\
\hline \multirow[t]{3}{*}{ Wind $(\mathrm{km} / \mathrm{h})$} & Max & $\max W$ & \\
\hline & Mean & meanW & \\
\hline & Min & $\min W$ & \\
\hline Precipitation $(\mathrm{mm})$ & Sum & precip & \\
\hline
\end{tabular}

4) Training, Validation, and Testing: For experiment purposes, the data sets are divided into three separate subgroups. They are training, validation and testing set. The training set is important where it is used to fit the prediction model, the validation set on the other hand is employed during model assessment and to prevent overfitting as well. Meanwhile, the testing set is where a real assessment is done to evaluate on of how well the prediction model generalize.

5) Performance Evaluation Metrics: To assess the Performance of identified hybrid algorithms, two statistical indicators were employed namely Theil's U and Root Mean Square Error (RMSPE). These criteria served as an interpreter in assessing the learning and generalization of identified prediction models. The formal definition of and Theil's U and RMSPE as shown in (9) and (10):

$$
\text { Theil's } U=\frac{\sqrt{\frac{1}{N} \sum_{n=1}^{N}\left(y_{n}-y\left(x_{n}\right)\right)^{2}}}{\sqrt{\frac{1}{N} \sum_{n=1}^{N}\left(y_{n}\right)^{2}+\sqrt{\frac{1}{N} \sum_{n=1}^{N}\left(y\left(x_{n}\right)\right)^{2}}}}
$$

$$
R M S P E=\sqrt{\frac{\left.\sum_{n=1}^{N}\left(\frac{y_{n}-y\left(x_{n}\right)}{y_{n}}\right)^{2}\right)}{N}}
$$

where

$n=1,2, \ldots . N$

$y_{n}=$ target values

$y\left(x_{n}\right)=$ predicted values

$N=$ Number of test data.

\section{RESULTS AND DISCUSSION}

The prediction model was realized on a real weather data in Kuala Lumpur. The data were collected from April 2016 to April 2017 as a complete time series with a time interval of 1 day.

By observing results recorded in Table 3, FA-LSSVM shows its superiority by recording the smallest value for both metrics, which are $0.0366 \%$ and 0.0172 respectively. These values are obtained by setting the $\gamma=49.1056$ and $\sigma 2=544.7772$. This is followed by DE-LSSVM by producing $0.0370 \%$ for RMSPE, and 0.0174 for Theil's U. On the other hand, MFO-LSSVM rank at third when the value of $\gamma$ is recorded to 1000 and $\sigma 2$ is 2.2962. Finally, CSA-LSSVM and ABC-LSSVM were on the fourth and last place respectively. By referring to both RMSPE and Theil's U, both values showed a good agreement.

In finding optimal values of the hyper-parameters of LSSVM, it is important to understand that too large or too small of the values may result in over-fitting or underfitting issue, which eventually will lead to poor generalization. Based on the table, the over-fitting issues can be seen occurred to MFO-LSSVM and ABC-LSSVM when the values of $\gamma$ for both hybrid algorithms reached the maximum value that has been set, which is 1000 . This situation contributes to the unsatisfied results for both MFO-LSSVM and ABC-LSSVM. 
TABLE III

MFO-LSSVM VS. CSA-LSSVM VS. ABC-LSSVM VS. FA-LSSVM VS. DELSSVM FOR WEATHER PREDICTION

\begin{tabular}{|c|c|c|c|c|}
\hline & Gam & Sig2 & RMSPE (\%) & Theils'U \\
\hline MFO-LSSVM & 1000 & 2.2962 & 0.0389 & 0.0183 \\
\hline CSA-LSSVM & 1.6475 & 12.4829 & 0.0413 & 0.0198 \\
\hline ABC-LSSVM & 1000 & 5.3542 & 0.0421 & 0.0204 \\
\hline FA-LSSVM & 49.1056 & 544.7772 & 0.0366 & 0.0172 \\
\hline DE-LSSVM & 16.1491 & 227.7176 & 0.0370 & 0.0174 \\
\hline
\end{tabular}

Meanwhile, Table 4 shows the prediction values by the identified hybrid algorithms during from day 363 to 372 (part of testing phase). The boldface values indicate the best performance on a respective day; the italic values represent the second best while the underlined values define the third best. Based on the listed values, it shows that the FALSSVM consistently be among the top 3 for most of the days.

TABLE IV

TARGET VS. MFO-LSSVM VS. CSA-LSSVM VS. ABC-LSSVM Vs. FALSSVM VS. DE-LSSVM

\begin{tabular}{|c|c|c|c|c|c|c|}
\hline Day & Target & $\begin{array}{c}\text { MFO- } \\
\text { LSSVM }\end{array}$ & $\begin{array}{c}\text { CSA- } \\
\text { LSSVM }\end{array}$ & $\begin{array}{c}\text { ABC- } \\
\text { LSSVM }\end{array}$ & $\begin{array}{c}\text { FA- } \\
\text { LSSVM }\end{array}$ & $\begin{array}{c}\text { DE- } \\
\text { LSSVM }\end{array}$ \\
\hline 363 & 28.0000 & 28.7326 & $\mathbf{2 8 . 2 7 7 8}$ & 28.5397 & $\underline{\mathbf{2 8 . 3 3 5 1}}$ & 28.2960 \\
\hline 364 & 28.0000 & 29.0686 & $\underline{28.9432}$ & 29.4053 & 27.8044 & $\mathbf{2 7 . 9 4 1 3}$ \\
\hline 365 & 28.0000 & 28.8042 & 28.5067 & 28.5354 & $\mathbf{2 8 . 3 5 4 3}$ & 28.4188 \\
\hline 366 & 28.0000 & 28.8278 & $\underline{28.2539}$ & 28.7269 & 28.1723 & $\mathbf{2 8 . 1 1 8 9}$ \\
\hline 367 & 28.0000 & 28.9960 & 28.8830 & $\underline{28.7778}$ & $\mathbf{2 8 . 4 4 1 9}$ & 28.4804 \\
\hline 368 & 28.0000 & $\underline{28.5275}$ & $\mathbf{2 8 . 1 7 0 5}$ & 28.2439 & 28.5407 & 28.5240 \\
\hline 369 & 29.0000 & 28.8898 & 28.5073 & $\mathbf{2 9 . 1 6 2 5}$ & $\underline{\mathbf{2 8 . 5 9 1 1}}$ & 28.5189 \\
\hline 370 & 29.0000 & $\mathbf{2 8 . 7 8 9 9}$ & $\underline{28.4412}$ & 28.6559 & 28.3908 & 28.4187 \\
\hline 371 & 29.0000 & 28.8676 & $\underline{29.0744}$ & 28.9515 & $\mathbf{2 9 . 0 2 0 2}$ & 29.0523 \\
\hline 372 & 29.0000 & 28.5214 & 28.5327 & 28.2336 & 28.6189 & $\mathbf{2 8 . 6 2 9 9}$ \\
\hline
\end{tabular}

Due to the superiority of FA-LSSVM over the other methods, a t-test was conducted to evaluate the statistical significance of the difference between the FA-LSSVM over the other identified algorithms. The t-test result is as tabulated in Table 5. From the recorded t-test value, it is demonstrated that the CSA-LSSVM, ABC-LSSVM, and DE-LSSVM are a good rival to the FA-LSSVM, except for MFO-LSSVM.

TABLE V

T-TEST

\begin{tabular}{|c|c|}
\hline Methods & T-test value \\
\hline FA-LSSVM - MFO-LSSVM & $2.802 \mathrm{E}-05$ \\
\hline FA-LSSVM - CSA-LSSVM & 0.2991 \\
\hline FA-LSSVM - ABC-LSSVM & 0.0636 \\
\hline FA-LSSVM - DE-LSSVM & 0.0165 \\
\hline
\end{tabular}

The illustration of the performance of the identified hybrid algorithms is depicted in Figure 7. From the figure, it is illustrated that most of the hybrid algorithms were incapable of producing an accurate prediction during the sudden ups and downs such as on day 380 and 410.

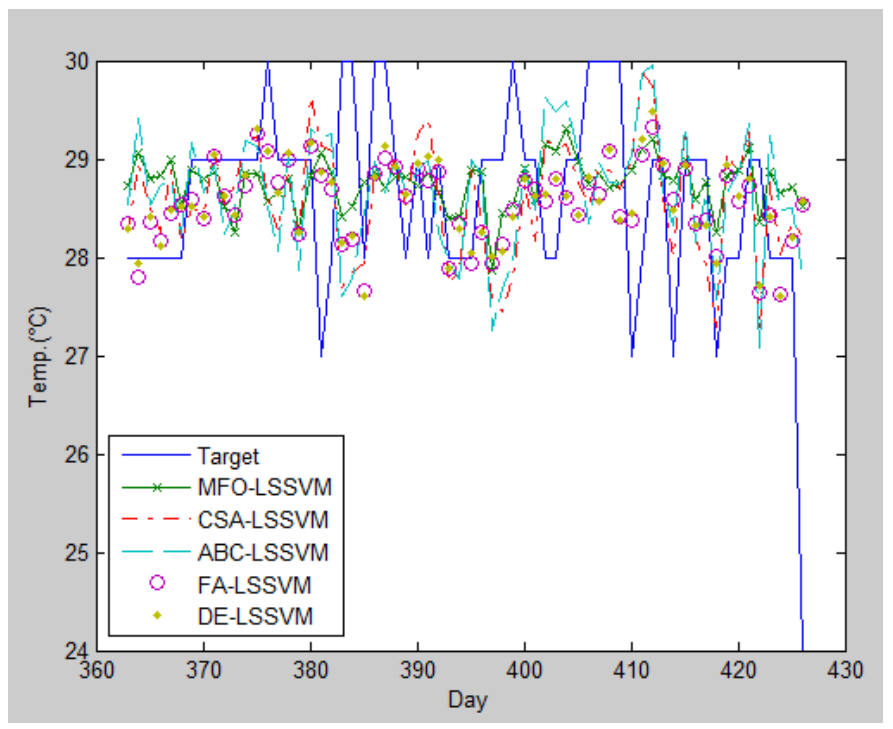

Fig. 7 MFO-LSSVM vs. CSA-LSSVM vs. ABC-LSSVM vs. FA-LSSVM vs. DE-LSSVM vs. Target

\section{CONCLUSION}

This paper provides a comparative study of MFO-LSSVM, CSA-LSSVM, ABC-LSSVM, FA-LSSVM, and DE-LSSVM for weather prediction. The experimental setup was designed for 5 days ahead prediction using daily data. Findings of the study demonstrate that these hybrid algorithms are suitable for the case under study, especially the FA-LSSVM. By achieving the smallest error rates, the FA-LSSVM outperform the other identified algorithms. Nonetheless, the capability of MFO-LSSVM, CSA-LSSVM, ABC-LSSVM, and DE-LSSVM could not be underestimated for being the great rival. For further work, this research could be enhanced by referring to various works in the literature which includes in [23]-[25].

\section{ACKNOWLEDGMENT}

The authors thank the Research Acculturation Grant Scheme (RAGS) for their financial support (Grant No.RDU151408).

\section{REFERENCES}

[1] Georgina Cosma, David Brown, Matthew Archer, Masood Khan, and A. G. Pockley, "A survey on computational intelligence approaches for predictive modeling in prostate cancer," Expert Systems with Applications, vol. 70, pp. 1-19, 2017.

[2] Monidipa Das and S. K. Ghosh, "Data-driven approaches for meteorological time series prediction: A comparative study of the state-of-the-art computational intelligence techniques," Pattern Recognition Letters, 2017.

[3] D. Petkovic, "Prediction of laser welding quality by computational intelligence approaches," Optik-International Journal for Light and Electron Optics, vol. 140, pp. 597-600, 2017.

[4] R. L. Haupt and S. E. Haupt, Practical Genetic Algorithms, Second ed. New Jersey: A John Wiley \& Sons, Inc., Publication, 2004.

[5] Kennedy and R. Eberhart, "Particle Swarm Optimization," in Proceedings of the IEEE International Conference on Neural Networks (ICNN), Australia, 1995, pp. pp. 1942-1948.

[6] M. Dorigo and T. Stutzle, Ant Colony Optimization. Cambridge: MIT Press 2004.

[7] S. Mirjalili, S. M. Mirjalili, and A. Lewis, "Grey Wolf Optimizer," Advances in Engineering Software, vol. 69, pp. 46-61, 2014. 
[8] Mohamad Shehab, A. T. Khader, and M. A. Al-Betar, "A survey on applications and variants of the cuckoo search algorithms," Applied Soft Computing, 2017.

[9] Georgina Cosma, David Browna, Matthew Archera, Masood Khanb, and A. G. Pockleyc, "A survey on computational intelligence approaches for predictive modeling in prostate cancer," Expert Systems with Applications, vol. 70, pp. 1-19, 2017.

[10] H. Son and C. Kim, "Short-term forecasting of electricity demand for the residential sector using weather and social variables," Resources, Conservation and Recycling, vol. 123, pp. 200-207, 2017.

[11] M. Wang, H. Chen, B. Yang, X. Zhao, H. Lufeng, Z. Cai, et al., "Toward an optimal kernel extreme learning machine using a chaotic moth-flame optimization strategy with applications in medical diagnoses," Neurocomputing, vol. 000, pp. 1-16, 2017.

[12] X. Wang, J. Wen, Y. Zhang, and Y. Wang, "Real estate price forecasting based on SVM optimized by PSO," Optik, vol. 125, pp. 1439-1443, 2014.

[13] D. Karaboga, "An Idea Based on Honey Bee Swarm for Numerical Optimization," Erciyes University, Technical ReportOctober, 2005 2005.

[14] J. A. K. Suykens, T. Van Gestel, J. De Brabanter, B. De Moor, and J. Vandewalle, Least Squares Support Vector Machines. Leuven, Belgium: World Scientific Publishing Co. Pte. Ltd., 2002.

[15] G.-B. Huang, H. Zhou, X. Ding, and R. Zhang, "Extreme Learning Machine for Regression and Multiclass Classification," IEEE Transactions on Systems, Man, and Cybernetics - Part B: Cybernetics, vol. 42, 2012.

[16] Q. Chen, Y. Wu, and X. Chen, "Research on Customers Demand Forecasting for E-business Web Site Based on LS-SVM," in Proceedings of the International Symposium on Electronic Commerce and Security, 2008, pp. 66-70.

[17] P. Ou and H. Wang, "Prediction of Stock Market Index Movement by Ten Data Mining Techniques," Modern Applied Science, vol. 3, pp. 28-42, 2009.
[18] M. Tarhouni, K. Laabidi, S. Zidi, and M. Ksouri-Lahmari, "A Nonlinear MIMO Systems Identification Based on Improved MultiKernel Least Squares Support Vector Machines (Improved MultiKernel LSSVM)," in Proceedings of the 8th International MultiConference on Systems, Signals and Devices, 2011.

[19] S. A. Bessedik and H. Hadi, "Prediction of Flashover Voltage of Insulators using Least Squares Support Vector Machine with Particle Swarm Optimization," Electric Power Systems Research, vol. 104, pp. 87-92, 2013.

[20] S. Mirjalili, "Moth-flame optimization algorithm: A novel natureinspired heuristic paradigm," Knowledge-Based Systems, vol. 89, pp. 228-249, 2015.

[21] X.-S. Yang, Nature-Inspired Metaheuristic Algorithms, Second ed. United Kingdom: Luniver Press, 2010.

[22] R. Storn and K. Price, "Differential Evolution- a simple and efficient heuristic for global optimization over continuous spaces," Journal of Global Optimization, vol. 11, pp. 341-359, 1997.

[23] M.A. Ismail, V. Mezhuyev, K. Moorthy, S. Kasim, A.O. Ibrahim, "Optimisation of Biochemical Systems Production using Hybrid of Newton Method, Differential Evolution Algorithm and Cooperative Coevolution Algorithm", Indonesian Journal of Electrical Engineering and Computer Science, vol.8, pp. 27-35, 2017.

[24] M.A. Ismail, V. Mezhuyev, S. Deris, M.S. Mohamad, S. Kasim, R.R. Saedudin, "Multi-objective Optimization of Biochemical System Production Using an Improve Newton Competitive Differential Evolution Method", International Journal on Advanced Science, Engineering and Information Technology, vol.7, pp.1535-1542, 2017.

[25] M.A. Ismail, S. Deris, M.S. Mohamad, M. A. Isa, A. Abdullah, M. A. Remli. S. M. Mohi-Aldeen, "A Hybrid of Optimization Method for Multi-Objective Constraint Optimization of Biochemical System Production", Journal of Theoretical and Applied Information Technology, vol.81, pp. 502-513, 2015. 